\title{
Dynamics of momentum entanglement in lowest-order QED
}

\author{
L. Lamata, ${ }^{1,2, *}$ J. León, ${ }^{1, \dagger}$ and E. Solano ${ }^{2,3, \$}$ \\ ${ }^{1}$ Instituto de Matemáticas y Física Fundamental, CSIC, Serrano 113-bis, 28006 Madrid, Spain \\ ${ }^{2}$ Max-Planck-Institut für Quantenoptik, Hans-Kopfermann-Strasse 1, D-85748 Garching, Germany \\ ${ }^{3}$ Sección Física, Departamento de Ciencias, Pontificia Universidad Católica del Perú, Apartado Postal 1761, Lima, Peru
}

(Received 7 September 2005; published 24 January 2006)

\begin{abstract}
We study the dynamics of momentum entanglement generated in the lowest-order QED interaction between two massive spin- $\frac{1}{2}$ charged particles, which grows in time as the two fermions exchange virtual photons. We observe that the degree of generated entanglement between interacting particles with initial well-defined momentum can be infinite. We explain this divergence in the context of entanglement theory for continuous variables, and show how to circumvent this apparent paradox. Finally, we discuss two different possibilities of transforming momentum into spin entanglement, through dynamical operations or through Lorentz boosts.
\end{abstract}

DOI: 10.1103/PhysRevA.73.012335

PACS number(s): 03.67.Mn, 12.20.-m, 03.65.Ud

\section{INTRODUCTION}

Entanglement is the physical property that Schrödinger described as Not one but the characteristic trait of quantum mechanics. It has played a fundamental role in the study of the completeness of quantum mechanics [1,2]. Nowadays, in quantum information theory, entanglement is considered as a physical resource, equivalent in many aspects to the role energy played in classical and quantum mechanics. Most applications in this field, like quantum teleportation [3], quantum communication [4], quantum cryptography [5], and some algorithms of quantum computation are carried out by using this intriguing quantum property [6]. A thorough study of entanglement in quantum information theory would demand a natural classification between discrete [7] and continuous variables [8].

In the past few years two apparently different fields, entanglement and relativity, have experienced intense research in an effort for treating them in a common framework [9-18]. Most of these works investigated the Lorentz covariance of entanglement through purely kinematic considerations, and only a few of them studied $a b$ initio the entanglement dynamics. For example, in the context of quantum electrodynamics (QED), Pachos and Solano [14] considered the generation and degree of entanglement of spin correlations in the scattering process of a pair of massive spin- $\frac{1}{2}$ charged particles, for an initially pure product state, in the low-energy limit and to the lowest order in QED. Manoukian and Yongram [18] computed the effect of spin polarization on correlations in a similar model, but also for the case of two photons created after $e^{+} e^{-}$annihilation, analyzing the violation of Bell's inequality [2]. In an earlier work, Grobe et al. [19] studied, in the nonrelativistic limit, the dynamics of entanglement in position and/or momentum of two electrons which interact with each other and with a nucleus via a smoothed Coulomb potential. They found that the associated

\footnotetext{
*Electronic address: lamata@imaff.cfmac.csic.es

†Electronic address: leon@imaff.cfmac.csic.es

†Electronic address: enrique.solano@mpq.mpg.de
}

quantum correlations manifest a tendency to increase as a function of the interaction time.

In this paper, we study to the lowest order in QED the interaction of a pair of identical, charged, massive spin- $\frac{1}{2}$ particles, and how this interaction increases the entanglement in the particle momenta as a function of time. We chose to work at lowest order, where entanglement already appears full-fledged, precisely for its simplicity. In particular, this allows us to set aside neatly other intricacies of QED, whose influence on entanglement should be the subject of separate analysis. Here, the generation of entanglement is a consequence of a conservation law: the total relativistic four momentum is preserved in the system evolution. This will also be the case in any interaction verifying this conservation law, as occurs in closed multipartite systems, while allowing the change in the individual momentum of each component. In the asymptotic limit, the infinite spacetime intervals involved in the $S$ matrix result in the generation of an infinite amount of entanglement for interacting particles with well-defined momentum. QED is a place where infinities can be avoided, and this will be also true, even though for other physical reasons, in the case of divergences appearing in momentum entanglement, a distinctive feature of continuous variables [8]. We will also discuss two different possibilities of establishing the transfer of entanglement between momentum and spin degrees of freedom in the collective two-particle system: through dynamical operations or Lorentz boosts.

In Sec. II, we analyze at lowest order and at finite time the generation of momentum entanglement between two electrons. In Sec. III, we calculate the Schmidt decomposition of the amplitude of a pair of spin- $\frac{1}{2}$ particles, showing the growth of momentum entanglement as they interact via QED. We obtain also analytic approximations of the Schmidt modes, both, in momentum and configuration spaces. In Appendix A, we include some notations and definitions related to entanglement theory for discrete and continuous variables. In Appendix B, we address the possibilities of transferring entanglement between momenta and spins via dynamical action, with local operations and classical communication (LOCC), or via kinematical action, with Lorentz transformations. 


\section{TWO ELECTRON GREEN FUNCTION IN PERTURBATION THEORY}

To address the properties of entanglement of a two electron system one needs the amplitude (wave function) $\psi\left(x_{1}, x_{2}\right)$ of the system, an object with 16 spinor components dependent on the configuration space variables $x_{1}, x_{2}$ of both particles. The wave functions were studied perturbatively by Bethe and Salpeter [20] and their evolution equation was also given by Gell-Mann and Low [21]. The wave function development is closely related to the two-particle Green function,

$$
K(1,2 ; 3,4)=\left(\Psi_{0}, T\left(\psi\left(x_{1}\right) \psi\left(x_{2}\right) \bar{\psi}\left(x_{3}\right) \bar{\psi}\left(x_{4}\right)\right) \Psi_{0}\right)
$$

which describes (in the Heisenberg picture) the symmetrized probability amplitude for one electron to proceed from event $x_{3}$ to event $x_{1}$ while the other proceeds from $x_{4}$ to $x_{2}$. If $u_{\mathrm{p} s}(3)$ describes the electron at 3 and $u_{\mathbf{p}^{\prime} s^{\prime}}(4)$ the one at 4 , then

$$
\begin{aligned}
\psi\left(x_{1}, x_{2}\right)= & \int d \sigma_{\mu}(3) d \sigma_{\nu}(4) K(1,2 ; 3,4) \gamma_{(3)}^{\mu} \gamma_{(4)}^{\nu} \\
& \times u_{\mathbf{p} s}(3) u_{\mathbf{p}^{\prime} s^{\prime}}(4),
\end{aligned}
$$

will be their correlated amplitude at 1,2 . In the free case this is just $u_{\mathbf{p} s}(1) u_{\mathbf{p}^{\prime} s^{\prime}}(2)$, but the interaction will produce a reshuffling of momenta and spins that may lead to entanglement. The two body Green function $K$ is precisely what we need for analyzing the dynamical generation of entanglement between both electrons.

Perturbatively [20],

$$
\begin{aligned}
K(1,2 ; 3,4)= & S_{F}(1,3) S_{F}(2,4)-e^{2} \int d^{4} x_{5} d^{4} x_{6} S_{F}(1,5) \\
& \times S_{F}(2,6) \gamma_{(5)}^{\mu} D_{F}(5,6) \gamma_{(6) \mu} S_{F}(5,3) S_{F}(6,4) \\
& +\cdots-1 \leftrightarrows 2,
\end{aligned}
$$

where all the objects appearing in the expansion are those of a free-field theory. We may call $K^{(n)}$ to the successive terms on the right-hand side of this expression. They will describe the transfer of properties between both particles due to the interaction. This reshuffling vanishes at lowest order, which gives just free propagation forward in time:

$$
\begin{gathered}
\int d^{3} x_{1} d^{3} x_{2} d^{3} x_{3} d^{3} x_{4} u_{\mathbf{p}_{1} s_{1}}^{\dagger}(1) u_{\mathbf{p}_{2} s_{2}}^{\dagger}(2) K^{(0)}(1,2 ; 3,4) \\
\quad \times \gamma_{(3)}^{0} u_{\mathbf{p}_{a} s_{a}}(3) \gamma_{(4)}^{0} u_{\mathbf{p}_{b} s_{b}}(4)=\theta\left(t_{1}-t_{3}\right) \theta\left(t_{2}-t_{4}\right) \\
\quad \times \delta_{s_{1} s_{a}} \delta_{s_{2} s_{b}} \delta^{(3)}\left(\mathbf{p}_{1}-\mathbf{p}_{a}\right) \delta^{(3)}\left(\mathbf{p}_{2}-\mathbf{p}_{b}\right)
\end{gathered}
$$

where $u_{\mathbf{p} s}(x)=(2 \pi)^{-3 / 2}(m / E)^{1 / 2} \exp (-i p x) u_{s}(\mathbf{p})$. The first effects of the interaction appear when setting $K^{(2)}$ instead of $K^{(0)}$ in the left-hand side of the above equation. The corresponding process is shown in Fig. 1.

To deal with this case we choose $t_{1}=t_{2}=t, t_{3}=t_{4}=-t$ and introduce the variables

$$
t_{+}=\frac{1}{2}\left(t_{5}+t_{6}\right), \quad t_{+} \in(-t, t)
$$

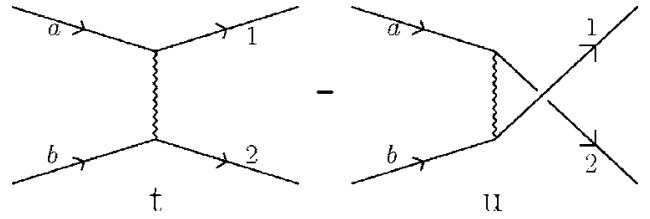

FIG. 1. Feynman diagrams for the QED interaction between two electrons (second order). The minus sign denotes the antisymmetry of the amplitude associated to the fermion statistics.

$$
t_{-}=\frac{1}{2}\left(t_{5}-t_{6}\right), \quad t_{-} \in\left[-\left(t-\left|t_{+}\right|\right), t-\left|t_{+}\right|\right],
$$

in Eq. (3), yielding

$$
\begin{aligned}
\tilde{K}^{(2)}(1,2 ; a, b ; t)= & \frac{2 i e^{2}}{(2 \pi)^{4}} \frac{j_{1 a}^{\mu} j_{\mu 2 b}}{\sqrt{2 E_{1} 2 E_{2} 2 E_{a} 2 E_{b}}} \\
& \times \delta^{(3)}\left(\mathbf{p}_{1}+\mathbf{p}_{2}-\mathbf{p}_{a}-\mathbf{p}_{b}\right) \\
& \times \int_{-\infty}^{\infty} \frac{d k^{0}}{\left(k^{0}\right)^{2}-\left(\mathbf{p}_{a}-\mathbf{p}_{1}\right)^{2}+i \epsilon} \\
& \times \int_{-t}^{t} d t_{+} e^{-i\left(E_{a}+E_{b}-E_{1}-E_{2}\right) t_{+}} \\
& \times \int_{-\left(t-\left|t_{+}\right|\right)}^{t-\left|t_{+}\right|} d t_{-} e^{-i\left(E_{1}-E_{2}+E_{b}-E_{a}+2 k^{0}\right) t_{-}} \\
& -\{1 \leftrightarrow 2\},
\end{aligned}
$$

where $\tilde{K}^{(2)}(1,2 ; a, b ; t)$ is a shorthand notation for what corresponds to (4) at second order, and $j_{k l}^{\mu}=\bar{u}_{k} \gamma^{\mu} u_{l}$. After some straightforward calculations, we obtain

$$
\begin{aligned}
\tilde{K}^{(2)}(1,2 ; a, b ; t)= & \frac{e^{2}}{4 \pi^{3}} \frac{\delta^{(3)}\left(\mathbf{p}_{1}+\mathbf{p}_{2}-\mathbf{p}_{a}-\mathbf{p}_{b}\right)}{\sqrt{2 E_{1} 2 E_{2} 2 E_{a} 2 E_{b}}} \times\left\{j _ { 1 a } ^ { \mu } j _ { \mu 2 b } \left[S_{t}(t)\right.\right. \\
& \left.\left.+\Upsilon_{t}(t)\right]-j_{2 a}^{\mu} j_{\mu 1 b}\left[S_{u}(t)+\Upsilon_{u}(t)\right]\right\}
\end{aligned}
$$

with

$$
\begin{aligned}
S_{t}(t)= & \frac{i}{\left(\frac{E_{1}-E_{2}+E_{b}-E_{a}}{2}\right)^{2}-\left(\mathbf{p}_{a}-\mathbf{p}_{1}\right)^{2}} \\
& \times \frac{\sin \left[\left(E_{1}+E_{2}-E_{a}-E_{b}\right) t\right]}{E_{1}+E_{2}-E_{a}-E_{b}}, \\
Y_{t}(t)= & \frac{1}{\left|\mathbf{p}_{a}-\mathbf{p}_{1}\right|} \times\{i] \frac{1}{\mu\left(\sum^{2}-\mu^{2}\right)} \\
& \left.+\frac{1}{\nu\left(\sum^{2}-\nu^{2}\right)}\right] \sum \sin \left(\sum t\right)-\left[\frac{1}{\sum^{2}-\mu^{2}}\right. \\
& \left.+\frac{1}{\sum^{2}-\nu^{2}}\right] \cos \left(\sum t\right)+\left[\frac{1}{\sum^{2}-\mu^{2}} e^{-i \mu t}\right. \\
& \left.\left.+\frac{1}{\sum^{2}-\nu^{2}} e^{-i \nu t}\right]\right\},
\end{aligned}
$$




$$
\begin{gathered}
\sum=E_{1}+E_{2}-E_{a}-E_{b}, \\
\mu=\Delta+2\left|\mathbf{p}_{a}-\mathbf{p}_{1}\right|, \\
\nu=-\Delta+2\left|\mathbf{p}_{a}-\mathbf{p}_{1}\right|, \\
\Delta=E_{1}-E_{2}+E_{b}-E_{a},
\end{gathered}
$$

and

$$
\begin{gathered}
S_{u}(t) \leftrightarrow S_{t}(t), \quad \Upsilon_{u}(t) \leftrightarrow \Upsilon_{t}(t), \\
1 \leftrightarrow 2 .
\end{gathered}
$$

$S_{t, u}$ are the only contributions that remain asymptotically ( $t$ $\rightarrow \infty$ ) leading to the standard scattering amplitude, while $\boldsymbol{Y}_{t, u}$ vanish in this limit. We recall that these are weak limits: no matter how large its modulus, the expression in Eq. (10) will vanish weakly due to its fast oscillatory behavior. On the other hand, the sinc function in Eq. (9) enforces energy conservation via

$$
\lim _{t \rightarrow \infty} \frac{\sin \left[\left(E_{1}+E_{2}-E_{a}-E_{b}\right) t\right]}{E_{1}+E_{2}-E_{a}-E_{b}}=\pi \delta\left(E_{1}+E_{2}-E_{a}-E_{b}\right) .
$$

This limit shows also that the entanglement in energies increases with time [22], see Appendix A, reaching its maximum (infinite) value when $t \rightarrow \infty$ for particles with initial well-defined momenta and energy. This result is independent of the chosen scattering configuration. Exact energy conservation at large times, united to a sharp momentum distribution of the initial states, would naturally result into a high degree of entanglement. The better defined the initial momentum of each electron, the larger the asymptotic entanglement. The physical explanation to this unbounded growth is the following: the particles with well-defined momentum (unphysical states) are spread over all space, and thus their interaction is ubiquitous, with the consequent unbounded degree of generated entanglement. This is valid for every experimental setup, except for those pathological cases where the amplitude cancels out, due to some symmetry. In the following section, and for illustrative purposes, we will single out these two possibilities.

(1) The case of an unbounded degree of attainable entanglement due to an incident electron with well defined momentum. We consider, with no loss of generality, a fuzzy distribution in momentum of the second initial electron, for simplicity purposes.

(2) Basically the same setup as in (1) but with a specific spin configuration, which leads to cancellation of the amplitude at large times due to symmetry, and thus to no asymptotic entanglement generation.

On the other hand, for finite times, nothing prevents a sizable contribution from Eq. (10). In fact, in the limiting case where $t^{-1}$ is large compared to the energies relevant in the problem, it may give the dominant contribution to entanglement. Whether the contribution from $\Upsilon_{t}(t)$ and $\Upsilon_{u}(t)$ is relevant, or not, depends on the particular case considered.

\section{TWO ELECTRON ENTANGLEMENT GENERATION AT LOWEST ORDER}

The electrons at $x_{3}, x_{4}$ will be generically described by an amplitude $F$

$$
\begin{aligned}
\psi_{F}\left(x_{3}, x_{4}\right)= & \sum_{s_{a}, s_{b}} \int d^{3} \mathbf{p}_{a} \int d^{3} \mathbf{p}_{b} F\left(\mathbf{p}_{a}, s_{a} ; \mathbf{p}_{b}, s_{b}\right) \\
& \times u_{\mathbf{p}_{a}, s_{a}}\left(x_{3}\right) u_{\mathbf{p}_{b}, s_{b}}\left(x_{4}\right)
\end{aligned}
$$

that should be normalizable to allow for a physical interpretation, i.e.,

$$
\sum_{s_{a}, s_{b}} \int d^{3} \mathbf{p}_{a} \int d^{3} \mathbf{p}_{b}\left|F\left(\mathbf{p}_{a}, s_{a} ; \mathbf{p}_{b}, s_{b}\right)\right|^{2}=1 .
$$

For separable states where $F(a ; b)=f_{a}\left(\mathbf{p}_{a}, s_{a}\right) f_{b}\left(\mathbf{p}_{b}, s_{b}\right), f_{a}$ and $f_{b}$ could be Gaussian amplitudes $g$ centered around a certain fixed momentum $\mathbf{p}^{0}$ and a certain spin component $s^{0}$,

$$
g(\mathbf{p}, s)=\frac{\delta_{s s^{0}}}{\left(\sqrt{\frac{\pi}{2}} \sigma\right)^{3 / 2}} \mathrm{e}^{-\left(\mathbf{p}-\mathbf{p}^{0}\right)^{2} / \sigma^{2}},
$$

which in the limit of vanishing widths give the standard, well-defined momentum state $\delta_{s s^{0}} \delta^{(3)}\left(\mathbf{p}-\mathbf{p}^{0}\right)$.

In the absence of interactions, a separable initial state will continue to be separable forever. However, interactions destroy this simple picture due to the effect of $U$. Clearly, the final state

$$
\begin{aligned}
F^{(2)}\left(\mathbf{p}_{1}, s_{1} ; \mathbf{p}_{2}, s_{2} ; t\right)= & \sum_{s_{a}, s_{b}} \int d^{3} \mathbf{p}_{a} \int d^{3} \mathbf{p}_{b} \widetilde{K}^{(2)}(1,2 ; a, b ; t) \\
& \times F\left(\mathbf{p}_{a}, s_{a} ; \mathbf{p}_{b}, s_{b}\right)
\end{aligned}
$$

cannot be factorized.

In the rest of this section we analyze the final state $F^{(2)}$ $\times\left(\mathbf{p}_{1}, s_{1} ; \mathbf{p}_{2}, s_{2} ; t\right)$ in Eq. (19) to show how the variables $\mathbf{p}_{1}$ and $\mathbf{p}_{2}$ get entangled by the interaction. We consider the nonrelativistic regime in which all intervening momenta and widths $\mathbf{p}, \sigma \ll m$, so the characteristic times $t$ under consideration are appreciable. We single out the particular case of a projectile fermion $a$ scattered off a fuzzy target fermion $b$ centered around $\mathbf{p}_{b}^{0}=0$. As a further simplification, we consider the projectile momentum sharply distributed around $\mathbf{p}_{a}^{0}$ $\left(\sigma_{a} \ll \mathbf{p}_{a}^{0}\right)$ so that the initial state can be approximated by

$$
F(a ; b) \approx \delta_{s_{a} s_{a}^{0}} \delta^{(3)}\left(\mathbf{p}_{a}-\mathbf{p}_{a}^{0}\right) \frac{\delta_{s_{b} s_{b}^{0}}}{\left(\sqrt{\frac{\pi}{2}} \sigma_{b}\right)^{3 / 2}} e^{-\left(\mathbf{p}_{b}-\mathbf{p}_{b}^{0}\right)^{2} / \sigma_{b}^{2}} .
$$

Our kinematical configuration would acquire complete generality should we introduce a finite momenta $\mathbf{p}_{b}^{0}$ for the initial electron $b$. The reference system would be in this case midway between the lab. system and the com. system. In short, the choice $\mathbf{p}_{b}^{0}=0$ will not affect the qualitative properties of entanglement generation. 


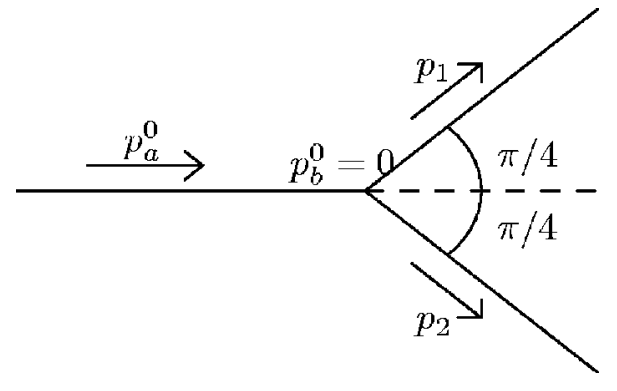

FIG. 2. Experimental setup considered in the calculations.

We will work in the lab frame, where particle $b$ shows a fuzzy momentum distribution around $\mathbf{p}_{b}^{0}=0$, and focus in the kinematical situation in which the final state momenta satisfy $\mathbf{p}_{1} \cdot \mathbf{p}_{2}=0$ and also $\mathbf{p}_{\alpha} \cdot \mathbf{p}_{a}^{0}=1 / \sqrt{2} p_{\alpha} p_{a}^{0}, \alpha=1,2$ (see Fig. 2). This choice not only avoids forward scattering divergencies but also simplifies the expression of the amplitude in Eq. (19), due to the chosen angles. For sure, the qualitative conclusions would also hold in other frames, like the center-ofmass one.

We obtain

$$
\begin{aligned}
E_{1}+ & E_{2}-E_{a}-E_{b} \mid \begin{array}{l}
\mathbf{p}_{b}=\mathbf{p}_{1}+\mathbf{p}_{2}-\mathbf{p}_{a}^{0} \\
\mathbf{p}_{a}=\mathbf{p}_{a}^{0}
\end{array} \\
& =\frac{p_{a}^{0}}{\sqrt{2} m}\left(p_{1}+p_{2}-\sqrt{2} p_{a}^{0}\right)+O\left(\left(p_{a}^{0} / m\right)^{3} p_{a}^{0}\right), \frac{\left(\mathbf{p}_{1}+\mathbf{p}_{2}-\mathbf{p}_{a}^{0}\right)^{2}}{\sigma^{2}} \\
& =\frac{\left(p_{1}-p_{a}^{0} / \sqrt{2}\right)^{2}}{\sigma^{2}}+\frac{\left(p_{2}-p_{a}^{0} / \sqrt{2}\right)^{2}}{\sigma^{2}},\left(\mathbf{p}_{1}-\mathbf{p}_{a}\right)^{2} \\
& =\left(p_{1}-p_{a}^{0} / \sqrt{2}\right)^{2}+\left(p_{a}^{0}\right)^{2} / 2,\left(\mathbf{p}_{2}-\mathbf{p}_{a}\right)^{2} \\
& =\left(p_{2}-p_{a}^{0} / \sqrt{2}\right)^{2}+\left(p_{a}^{0}\right)^{2} / 2 .
\end{aligned}
$$

Here, boldface characters represent trivectors, otherwise they represent their associated norms. We perform now the following change of variables,

$$
\frac{p}{\sqrt{2}}=\frac{1}{\sigma}\left(p_{1}-\frac{p_{a}^{0}}{\sqrt{2}}\right), \frac{q}{\sqrt{2}}=\frac{1}{\sigma}\left(p_{2}-\frac{p_{a}^{0}}{\sqrt{2}}\right),
$$

turning the amplitude in Eq. (19) into

$$
\begin{aligned}
& F^{(2)}\left(p, s_{1} ; q, s_{2} ; t\right) \propto \frac{\sin [(p+q) \tilde{t}]}{\tilde{\Sigma}} \times\left[\frac{\left(j_{1 a}^{\mu} j_{\mu 2 b}\right)_{s_{b}=s_{b}^{0}}^{s_{a}=s_{a}^{0}}}{p^{2}+\left(\frac{p_{a}^{0}}{\sigma}\right)^{2}}-\frac{\left(j_{1 b}^{\mu} j_{\mu 2 a}\right)_{s_{b}=s_{b}}^{s_{a}=s_{a}^{0}}}{q^{2}+\left(\frac{p_{a}^{0}}{\sigma}\right)^{2}}\right] e^{-p^{2} / 2} e^{-q^{2} / 2}+\left(\frac { ( j _ { 1 a } ^ { \mu } j _ { \mu 2 b } ) _ { s _ { b } = s _ { b } ^ { 0 } } ^ { s _ { a } = s _ { a } ^ { 0 } } } { \tilde { \mu } / 2 } \left\{-\frac{1}{\tilde{\mu}\left(\tilde{\Sigma}^{2}-\tilde{\mu}^{2}\right)}\right.\right. \\
& \left.\times \tilde{\Sigma} \sin [(p+q) \tilde{t}]-\frac{i}{\tilde{\Sigma}^{2}-\tilde{\mu}^{2}}\left\{\cos [(p+q) \tilde{t}]-\mathrm{e}^{-i\left(2 m / p_{a}^{0}\right) \tilde{\mu} \tilde{t}}\right\}-\{p, 1 \leftrightarrow q, 2\}\right) e^{-p^{2} / 2} e^{-q^{2} / 2},
\end{aligned}
$$

where $\quad \widetilde{\Sigma}=p_{a}^{0} /(2 m)(p+q), \quad \tilde{\mu}=\sqrt{2} \sqrt{p^{2}+\left(p_{a}^{0} / \sigma\right)^{2}}, \quad$ and $\tilde{t} \equiv p_{a}^{0} \sigma /(2 m) t$. In the following, we analyze different specific spin configurations in the nonrelativistic limit with the help of Eq. (23). We consider an incident particle energy of around $1 \mathrm{eV} \ll m\left(p_{a}^{0}=1 \mathrm{KeV}\right)$, and a momentum spreading $\sigma$ one order of magnitude less than $p_{a}^{0}$. We make this choice of $p_{a}^{0}$ and $\sigma$ to obtain longer interaction times, of femtoseconds $\left(t=\left(2 m /\left(p_{a}^{0} \sigma\right) \widetilde{t}\right.\right.$. Thus the parameter values we consider in the subsequent analysis are $p_{a}^{0} / m=0.002$ and $\sigma / m$ $=0.0002$. We consider the initial spin state for particles $a$ and $b$ as

$$
\left|s_{a}^{0} s_{b}^{0}\right\rangle=|\uparrow \downarrow\rangle,
$$

along an arbitrary direction that will serve to measure spin components in all the calculations. The physical results we are interested in do not depend on this choice of direction. The QED interaction, in the nonrelativistic regime considered, at lowest order, is a Coulomb interaction that does not change the spins of the fermions. In fact, $\left(j_{1 a}^{\mu} j_{\mu 2 b}\right)$ $\simeq 4 m^{2} \delta_{s_{a}^{0} s_{1}} \delta_{s_{b}^{0} s_{2}}, \quad\left(j_{1 b}^{\mu} j_{\mu 2 a}\right) \simeq 4 m^{2} \delta_{s_{b}^{0} s_{1}} \delta_{s_{a}^{0} s_{2}}$. Given the initial spin states of Eq. (24), depending on whether the channel is $t$ or $u$, the possible final spin states are

$$
\begin{aligned}
& \left|s_{1} s_{2}\right\rangle_{t}=|\uparrow \downarrow\rangle, \\
& \left|s_{1} s_{2}\right\rangle_{u}=|\downarrow \uparrow\rangle .
\end{aligned}
$$

Due to the fact that the considered fermions are identical, the resulting amplitude after applying the Schmidt procedure is a superposition of Slater determinants [23-25]. Whenever this decomposition contains just one Slater determinant (Slater number equal to 1) the state is not entangled: its correlations are just due to the statistics and are not useful for the applications because they do not contain any additional physical information. If the amplitude contains more than one determinant, the state is entangled. Splitting the amplitude in the corresponding ones for the $t$ and $u$ channels, we have 

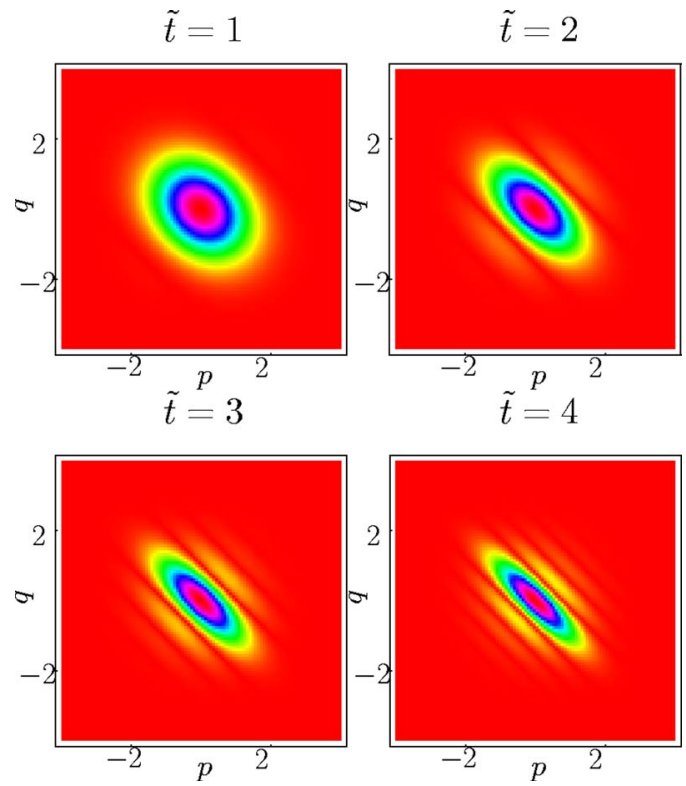

FIG. 3. (Color online) $\left|F^{(2)}(p, \uparrow ; q, \downarrow ; t)_{t}\right|$ versus $p, q$ at $\tilde{t}$ $=1,2,3,4$.

$$
\begin{aligned}
& F^{(2)}(p, \uparrow ; q, \downarrow ; t)_{t} \propto \frac{\sin [(p+q) \tilde{t}]}{\tilde{\Sigma}} \frac{1}{p^{2}+\left(\frac{p_{a}^{0}}{\sigma}\right)^{2}} e^{-p^{2} / 2} e^{-q^{2} / 2} \\
& +\frac{1}{\tilde{\mu} / 2}\left(-\frac{1}{\tilde{\mu}\left(\tilde{\Sigma}^{2}-\tilde{\mu}^{2}\right)} \tilde{\sum} \sin [(p+q) \tilde{t}]\right. \\
& \left.-\frac{i}{\tilde{\Sigma}^{2}-\tilde{\mu}^{2}}\left\{\cos [(p+q) \tilde{t}]-e^{-i\left(2 m / p_{a}^{0}\right) \tilde{\mu} \tilde{t}}\right\}\right) \\
& \times e^{-p^{2} / 2} e^{-q^{2} / 2},
\end{aligned}
$$

with

$$
\begin{aligned}
F^{(2)}(p, \downarrow ; q, \uparrow ; t)_{u} & \leftrightarrow F^{(2)}(p, \uparrow ; q, \downarrow ; t)_{t}, \\
p & \leftrightarrow q .
\end{aligned}
$$

In the infinite time limit the sinc function converges to $\delta(p+q)$, which is a distribution with infinite entanglement [22]. The presence of the sinc function is due to the finite time interval of integration in Eq. (7). This kind of behavior can be interpreted as a time diffraction phenomenon [26]. It has direct analogy with the diffraction of electromagnetic waves that go through a single slit of width $2 L$ comparable to the wavelength $\lambda$. The analogy is complete if one identifies $\tilde{t}$ with $L$ and $p+q$ with $2 \pi / \lambda$.

In Fig. 3, we plot the modulus of Eq. (27) versus $p$, $q$, at times $\tilde{t}=1,2,3,4$. This graphic shows the progressive clustering of the amplitude around the curve $q=-p$, due to the function $\sin [(p+q) \tilde{t}] /(p+q)$. This is a clear signal of the growth in time of the momentum entanglement. Figure 3 puts also in evidence the previously mentioned time diffraction pattern.
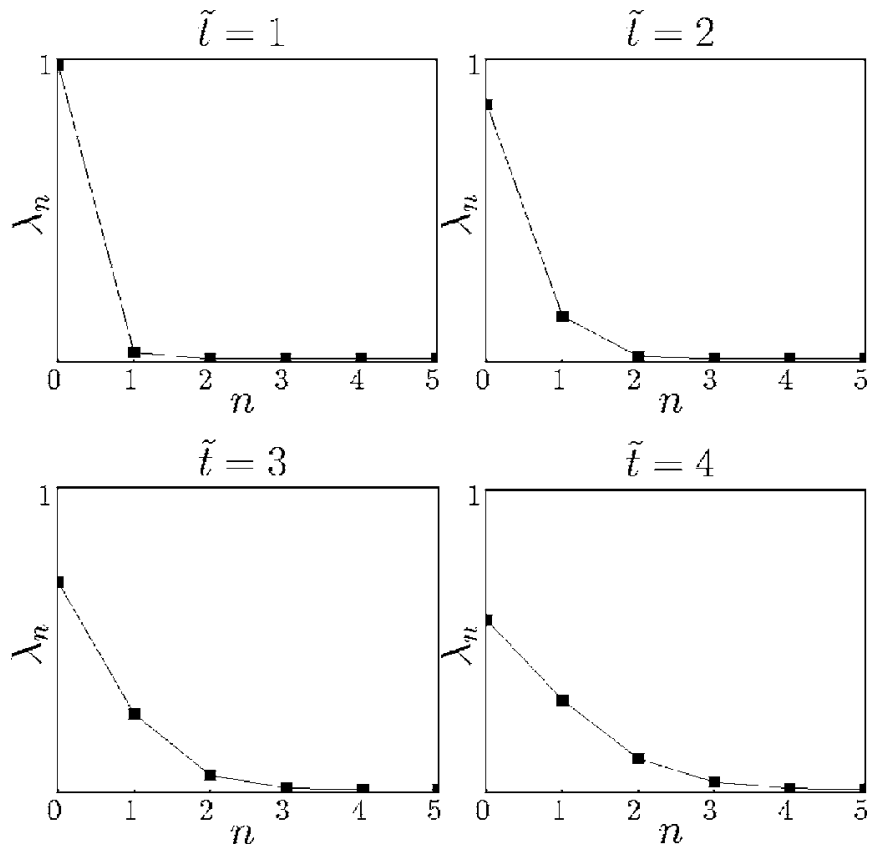

FIG. 4. Eigenvalues $\lambda_{n}$ versus $n$ at times $\tilde{t}=1,2,3,4$.

We have applied the method for obtaining Schmidt decomposition given in Ref. [22] to Eq. (27), considering for the orthonormal functions $\left\{O^{(1)}(p)\right\},\left\{O^{(2)}(q)\right\}$ Hermite polynomials with their weights, to take advantage of the two Gaussian functions. We obtain the Schmidt decomposition for $\tilde{t}=1,2,3,4$, where the error with matrices $C_{m n} 12 \times 12$ or smaller is $d_{m_{0}, n_{0}}^{2} \leqslant 7 \times 10^{-3}$ in all considered cases. We plot in Fig. 4 the coefficients $\lambda_{n}$ of the Schmidt decomposition of Eq. (27) as a function of $n$, for times $\tilde{t}=1,2,3,4$. The number of $\lambda_{n}$ different from zero increases as time is elapsed, and thus the entanglement grows.

The complete the Schmidt decomposition, including channels $t$ and $u$, is given in terms of Slater determinants [23], and is usually called Slater decomposition. It is obtained antisymmetrizing the amplitude for channel $t$

$$
\begin{aligned}
& F^{(2)}\left(p, s_{1} ; q, s_{2} ; t\right) \\
& \propto \sum_{n} \sqrt{\lambda_{n}(\widetilde{t})} \\
& \quad \times \frac{\psi_{n}^{(1)}(p, \widetilde{t})|\uparrow\rangle \psi_{n}^{(2)}(q, \widetilde{t})|\downarrow\rangle-\psi_{n}^{(2)}(p, \widetilde{t})|\downarrow\rangle \psi_{n}^{(1)}(q, \widetilde{t})|\uparrow\rangle}{\sqrt{2}},
\end{aligned}
$$

where the modes $\psi_{n}^{(1)}(k, \widetilde{t})$ and $\psi_{n}^{(2)}(k, \widetilde{t})$ are the Schmidt modes of the channel $t$ obtained for particles 1 and 2, respectively, and they correspond to the modes of the channel $u$ for particles 2 and 1, respectively.

A measure of the entanglement of a pure bipartite state of the form of Eq. (29), equivalent to the entropy of entanglement $S$, is given by the Slater number [19]

$K$ gives the number of effective Slater determinants which appear in a certain pure bipartite state in the form of Eq. (29). The larger the value of $K$, the larger the entanglement. For 


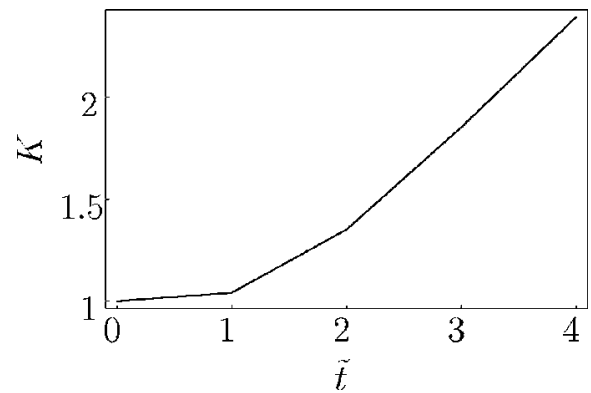

FIG. 5. Slater number $K$ as a function of the elapsed time $\tilde{t}$.

$K=1$ (one Slater determinant) there is no entanglement. This measure is obtained considering the average probability, which is given by $\sum_{n=0}^{\infty} \lambda_{n}^{2}\left(\sum_{n=0}^{\infty} \lambda_{n}=1\right.$, and thus $\left\{\lambda_{n}\right\}$ can be seen as a probability distribution). The inverse of the average probability is the Slater number. Its attractive properties are that it is independent of the representation of the wave function, it is gauge invariant, and it reaches its minimum value of 1 for the separable state (single Slater determinant). In Fig. 5, we show the Slater number $K$ as a function of elapsed time $\tilde{t}$, verifying that the entanglement increases as the system evolves. It can be appreciated in this figure the monotonic growth of entanglement, due to the fact that we have considered an incident electron with a well-defined momentum. In realistic physical situations with wave packets, this growth would stop, due to the momentum spread of the initial electrons. The general trend is that the higher the precision in the incident electron momentum, the larger the resulting asymptotic entanglement. The fact that the entanglement in momenta between the two fermions increases with time is a consequence of the interaction between them. We remark that the entanglement cannot grow unless the two particles "feel" each other. The correlations in momenta are not specific of QED: the effect of any interaction producing momentum exchange while conserving total momentum will translate into momentum correlations.

The Schmidt modes in momenta space for the amplitude of Eq. (27) are given by

$$
\begin{gathered}
\psi_{m}^{(\alpha)}(k, \widetilde{t}) \simeq e^{-k^{2} / 2} \sum_{n=0}^{n_{0}}\left(\sqrt{\pi} 2^{n} n !\right)^{-1 / 2} A_{m n}^{(\alpha)}(\widetilde{t}) H_{n}(k), \\
\alpha=1,2,
\end{gathered}
$$

where $n_{0}$ is the corresponding cut-off and the values of the coefficients $A_{m n}^{(\alpha)}(\widetilde{t})$ are obtained through the method given in Ref. [22]. The modes in momenta space depend on time because they are not stationary states: the QED dynamics between the two fermions and the indeterminacy on the energy at early stages of the interaction give this dependence. By construction, the coefficients $A_{m n}^{(\alpha)}(\widetilde{t})$ do not depend on $p$, $q$.

We plot in Fig. 6 the Schmidt modes $\psi_{n}^{(1)}(p, \widetilde{t})$ at times $\tilde{t}$ $=1,2,3,4$ for $n=0,1,2,3$ [we are plotting specifically the real part of each mode only, which approximates well the whole mode, because Eq. (27) is almost real for the cases considered]. The sharper modes for each $n$ correspond to the

$$
n=0
$$

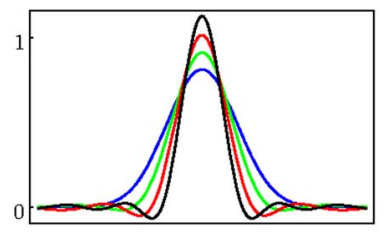

$n=2$
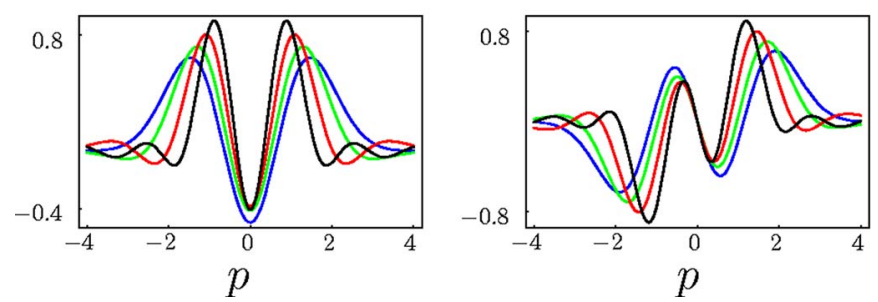

FIG. 6. (Color online) Schmidt modes $\psi_{n}^{(1)}(p, \widetilde{t})$ at times $\tilde{t}$ $=1,2,3,4$ for $n=0,1,2,3$. The sharper modes for each $n$ correspond to the later times.

later times. Each Schmidt mode is well approximated at early times by the corresponding Hermite orthonormal function, and afterwards it sharpens and deviates from that function: it gets corrections from higher-order polynomials. The fact that the modes get thinner with time is related to the behavior of Eq. (27) at large times. In particular the sinc function goes to $\delta(p+q)$ and thus the amplitude gets sharper.

Now we consider the Schmidt modes in configuration space. To obtain them, we just Fourier transform the modes of Eq. (31) with respect to the momenta $p_{1}, p_{2}$

$$
\widetilde{\psi}_{m}^{(\alpha)}\left(x_{\alpha}, \widetilde{t}\right)=\frac{1}{\sqrt{2 \pi}} \int_{-\infty}^{\infty} d p_{\alpha} e^{i\left(p_{\alpha} x_{\alpha}-p_{\alpha}^{2} / 2 m t\right)} \psi_{m}^{(\alpha)}\left(k\left(p_{\alpha}\right), \widetilde{t}\right)
$$

where $\alpha=1,2$. The dependence of $p$ on $p_{1}$ and $q$ on $p_{2}$ is given through Eq. (22). The factor $e^{-i p_{\alpha}^{2} / 2 m t}$ in Eq. (32) is the one which commutes the states between the interaction picture (considered in Eq. (31) and in the previous calculations in Secs. II and III) and the Schrödinger picture. [27]:

The Hermite polynomials obey the following expression

$$
\int_{-\infty}^{\infty} d x e^{-(x-y)^{2}} H_{n}(\alpha x)=\sqrt{\pi}\left(1-\alpha^{2}\right)^{n / 2} H_{n}\left(\frac{\alpha y}{\sqrt{1-\alpha^{2}}}\right) .
$$

With the help of Eq. (33) and by linearity of the Fourier transforms, we are able to obtain analytic expressions for the Schmidt modes in configuration space (to a certain accuracy, which depends on the cut-offs considered). This is possible because the dispersion relation of the massive fermions in the considered nonrelativistic limit is $E_{\alpha}=p_{\alpha}^{2} / 2 m$, and thus the integral of Eq. (32) can be obtained analytically using Eq. (33). 
The corresponding Schmidt modes in configuration space are then given by

$$
\widetilde{\psi}_{m}^{(\alpha)}\left(\widetilde{x}_{\alpha}, \widetilde{t}\right) \simeq \sum_{n=0}^{n_{0}} A_{m n}^{(\alpha)}(\widetilde{t}) \widetilde{O}_{n}^{(\alpha)}\left(\widetilde{x}_{\alpha}, \widetilde{t}\right), \quad \alpha=1,2,
$$

where the orthonormal functions in configuration space are

$$
\begin{aligned}
\widetilde{O}_{n}^{(\alpha)}\left(\widetilde{x}_{\alpha}, \widetilde{t}\right)= & i^{n}\left(\sqrt{\pi} 2^{n} n !\right)^{-1 / 2} \frac{e^{-i n \arctan (\widetilde{\sigma} t)+i \widetilde{\sigma}^{-1}\left(\tilde{x}_{\alpha}-\tilde{t} / 2\right)}}{\sqrt{1+i \widetilde{\sigma}}} \\
& \times e^{-\frac{\left(\widetilde{x}_{\alpha}-\widetilde{t}\right)^{2}}{2(1+i \widetilde{\tau})}} H_{n}\left[\frac{\widetilde{t}-\tilde{x}_{\alpha}}{\sqrt{1+(\widetilde{\sigma})^{2}}}\right] .
\end{aligned}
$$

In Eqs. (34) and (35), we are using dimensionless variables, $\tilde{x}_{\alpha}=\sigma x_{\alpha} / \sqrt{2}, \alpha=1,2, \tilde{\sigma}=\sigma / p_{a}^{0}$, and the dimensionless time defined before, $\tilde{t}=p_{a}^{0} \sigma /(2 m) t$. The modes in Eqs. (34) and (35) are normalized in the variables $\tilde{x}_{\alpha}$. The orthonormal functions of Eq. (35) propagate in space at a speed $p_{a}^{0} / \sqrt{2} m$ and they spread in their evolution. Additionally, the modes of Eq. (34) have also the time dependence of $A_{m n}^{(\alpha)}(\widetilde{t})$. The Slater decomposition in configuration space, obtained Fourier transforming the modes of Eq. (31) is

$$
\begin{aligned}
\widetilde{F}^{(2)}\left(\widetilde{x}_{1}, \widetilde{x}_{2}, \widetilde{t}\right) \propto & \sum_{n} \sqrt{\frac{\lambda_{n}(\widetilde{t})}{2}}\left[\widetilde{\psi}_{n}^{(1)}\left(\widetilde{x}_{1}, \widetilde{t}\right)|\uparrow\rangle \widetilde{\psi}_{n}^{(2)}\left(\widetilde{x}_{2}, \widetilde{t}\right)|\downarrow\rangle-\widetilde{\psi}_{n}^{(2)}\right. \\
& \left.\times\left(\widetilde{x}_{1}, \widetilde{t}\right)|\downarrow\rangle \widetilde{\psi}_{n}^{(1)}\left(\widetilde{x}_{2}, \widetilde{t}\right)|\uparrow\rangle\right] .
\end{aligned}
$$

The coefficients $\lambda_{n}(\widetilde{t})$ are unaffected by the Fourier transformation, and thus the degree of entanglement in configuration space is the same as in momenta space.

We consider now the initial spin configuration

$$
\left|s_{a}^{0} s_{b}^{0}\right\rangle=|\uparrow \uparrow\rangle,
$$

where the only possible final state in the nonrelativistic limit is

$$
\left|s_{1} s_{2}\right\rangle=|\uparrow \uparrow\rangle .
$$

In this case, the sinc term goes to zero, because the momentum part of this term is antisymmetric in $p^{2}, q^{2}$ and the sinc function goes to $\delta(p+q)$, which has support (as a distribution) on $q=-p$. We point out that the sinc contribution to this amplitude is negligible because of the particular setup chosen. In other experiment configurations the amplitude in Eq. (19) associated to the spin states of Eqs. (37) and (38) would have appreciable sinc term and thus increasing momenta entanglement with time. On the other hand, in this case the contribution from $\Upsilon_{t}(t)$ in Eq. (10) and $\Upsilon_{u}(t)$ is even smaller than the sinc term, and converges weakly to zero.

We plot in Fig. 7 the real and imaginary parts of the term associated to $Y_{t}(t)$ and $\Upsilon_{u}(t)$ in Eq. (23), which we denote by $g(p, q, \widetilde{t})$, for spin states of Eqs. (37) and (38) as a function of time $\tilde{t} \in(1,1.001)$ and having $p=1, q=1.2$. We want to show with it the strong oscillatory character of the amplitude with time, and how all the contributions interfere destructively with each other giving a zero final value. This is similar to the stationary phase procedure, in which only the contributions in proximity to the stationary value of the phase do

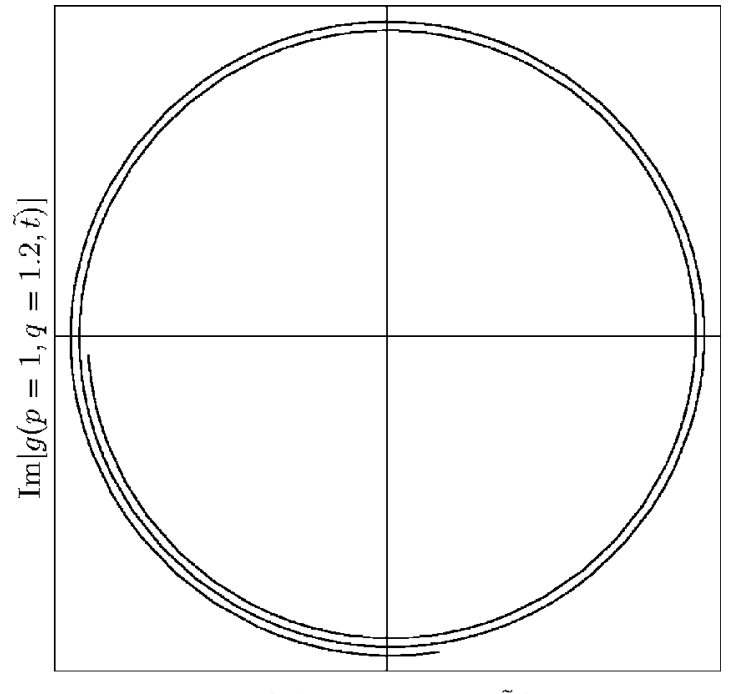

$\operatorname{R} c .[g(p=1, q=1.2, \tilde{t})]$

FIG. 7. Real and imaginary parts of the amplitude $g(p, q, \widetilde{t})$ for $p=1, q=1.2$, and $\tilde{t} \in(1,1.001)$ (arbitrary units).

interfere constructively and are appreciable. What we display here is the weak convergence to zero for the functions $\Upsilon_{t}(t)$ and $\Upsilon_{u}(t)$.

In this section, we investigated the generation of entanglement in momenta between two identical spin- $\frac{1}{2}$ particles which interact via QED. We showed how the correlations grow as the energy conservation is increasingly fulfilled with time. The previous calculation had, however, the approximation of considering a projectile particle with a perfectly welldefined momentum, something not achievable in practice. This is a first step towards a real experiment, where both fermions will have a dispersion in momenta and thus infinite entanglement will never be reached, due to the additional integrals of the Dirac delta $\delta(\Delta E)$ over the momentum spread.

\section{CONCLUSIONS}

We analyzed the dynamical generation of entanglement between two electrons due to their mutual interaction by means of the Slater number. In the asymptotic limit, and for electrons whose initial momenta are sharply defined, entanglement divergencies may appear. We observe that considering finite-time intervals of interaction, and/or a certain spreading in the particles momentum, the entanglement remains finite. We have studied the dynamical generation of momentum entanglement of two spin-1/2 particles at lowest-order QED, observing how the correlations increase as the particles exchange virtual photons. We obtain the Schmidt decomposition of the scattering amplitude, given in terms of Slater determinants, and the Slater number, which clearly shows the growth of entanglement with time. We also obtain analytic approximations of the Schmidt modes, both in momentum and configuration spaces.

\section{ACKNOWLEDGMENTS}

J.L. thanks I. Bialynicki-Birula for useful discussions and private communications. L.L. thanks I. Cirac for hospitality 
at the Max-Planck Institute for Quantum Optics and acknowledges financial support from FPU Grant No. AP20030014. The work of J.L. and L.L. was partially supported by the Spanish Ministerio de Educación y Ciencia under Project No. BMF 2002-00834. E.S. acknowledges financial support from the EU Project RESQ.

\section{APPENDIX A: ENTANGLEMENT IN FINITE AND INFINITE DIMENSIONAL HILBERT SPACES}

We consider a composite system $\mathcal{S}$ described by a Hilbert space $\mathcal{H}$, which may be either finite or infinite dimensional. This space is constructed as the tensor product of the Hilbert spaces associated to each of the subsystems, $\mathcal{S}_{\alpha}$, of $\mathcal{S}$. For simplicity, and in view of our present purposes, we restrict ourselves to pure states of a bipartite system $\mathcal{S}$. Thus, $\alpha$ $=1,2$, and $\mathcal{H}=\mathcal{H}_{1} \otimes \mathcal{H}_{2}$.

Definition: product state. A vector state $|\Psi\rangle$ of the system $\mathcal{S}$ is a product state if it can be written as

$$
|\Psi\rangle=\left|\Psi^{(1)}\right\rangle\left|\Psi^{(2)}\right\rangle,
$$

where $\left|\Psi^{(1)}\right\rangle \in \mathcal{H}_{1}$ and $\left|\Psi^{(2)}\right\rangle \in \mathcal{H}_{2}$.

Definition: entangled state. A vector state $|\Psi\rangle$ of the system $\mathcal{S}$ is entangled if it is not a product state. A relevant example, where $\operatorname{dim}\left(\mathcal{H}_{1}\right)=\operatorname{dim}\left(\mathcal{H}_{2}\right)=2$, is called the singlet state,

$$
\left|\Psi^{-}\right\rangle=\frac{1}{\sqrt{2}}\left(\left|\Psi_{1}^{(1)}\right\rangle\left|\Psi_{2}^{(2)}\right\rangle-\left|\Psi_{2}^{(1)}\right\rangle\left|\Psi_{1}^{(2)}\right\rangle\right) .
$$

A very useful tool for analyzing the entanglement of pure states of bipartite systems is given by the Schmidt decomposition $[28,29]$. It basically consists in expressing the pure bipartite state as a sum of biorthonormal products, with positive coefficients $\sqrt{\lambda_{n}}$, as follows:

$$
|\Psi\rangle=\sum_{n=0}^{d-1} \sqrt{\lambda_{n}}\left|\Psi_{n}^{(1)}\right\rangle\left|\Psi_{n}^{(2)}\right\rangle,
$$

where $\left\{\left|\Psi_{n}^{(1)}\right\rangle\right\},\left\{\left|\Psi_{n}^{(2)}\right\rangle\right\}$, are orthonormal bases associated to $\mathcal{H}_{1}$ and $\mathcal{H}_{2}, \quad$ respectively. In Eq. (A3), $d$ $=\min \left\{\operatorname{dim}\left(\mathcal{H}_{1}\right), \operatorname{dim}\left(\mathcal{H}_{2}\right)\right\}$, and it may be infinite, as for in continuous variable systems, describing momentum, energy, position, frequency, or the like. In these cases, the states $\left|\Psi_{n}^{(\alpha)}\right\rangle$ would be (square integrable) $L^{2}$ wave functions,

$$
\left\langle p \mid \Psi_{n}^{(\alpha)}\right\rangle=\psi_{n}^{(\alpha)}(p), \quad \alpha=1,2,
$$

where $p$ denotes the corresponding continuous variable.

For pure bipartite states a relevant measure of entanglement is the entropy of entanglement, $S$. Given a state $|\Psi\rangle$, it is defined as the von Neumann entropy of the reduced density matrix with respect to $S_{1}$ or $S_{2}$,

$$
S=-\sum_{n=0}^{d-1} \lambda_{n} \log _{2} \lambda_{n},
$$

where the $\lambda_{n}$ 's are given in Eq. (A3). In general, $S \geqslant 0, S$ $=0$ for a product state, and the more entangled a state is, the larger $S$. For a maximally entangled state, $S=\log _{2} d$, and if $d=\infty$, then $S$ diverges.
An interesting work where the Schmidt decomposition for continuous variables is analyzed discretizing the corresponding integral equations can be found in Ref. [29]. Another method for obtaining the continuous variables Schmidt decomposition, based in decomposing the bipartite wave function in complete sets of orthonormal functions, is developed in Ref. [22].

\section{APPENDIX B: ENTANGLEMENT TRANSFER BETWEEN MOMENTUM AND SPIN}

\section{Dynamical transfer and distillation}

In Sec. III we computed the entanglement in momenta for a pair of identical spin- $\frac{1}{2}$ particles which interact through the exchange of a virtual photon. The sharper the initial momentum distribution of the incident fermion, and the longer the interaction time, the larger the entanglement in momenta. Heisenberg's principle, on the other hand, establishes a limit to the precision with which the momentum may be defined and hence to the achievable degree of entanglement.

It is possible, in principle, to transform the entanglement in momenta into entanglement in spins. This is easily seen in terms of the majorization criterion $[6,30]$, which is of practical interest because the experimentalist usually manipulates spins. Here, we will analyze this entanglement transfer.

Majorization is an area of mathematics which predates quantum mechanics. Quoting Nielsen and Chuang, "Majorization is an ordering on $d$-dimensional real vectors intended to capture the notion that one vector is more or less disordered than another." We consider a pair of $d$-dimensional vectors, $x=\left(x_{1}, \ldots, x_{d}\right)$ and $y=\left(y_{1}, \ldots, y_{d}\right)$. We say $x$ is majorized by $y$, written $x<y$, if $\sum_{j=1}^{k} x_{j}^{\downarrow} \leqslant \sum_{j=1}^{k} y_{j}^{\downarrow}$ for $k=1, \ldots, d$, with equality instead of inequality for $k=d$. We denote by $z^{\downarrow}$ the components of $z$ in decreasing order $\left(z_{1}^{\downarrow} \geqslant z_{2}^{\downarrow} \geqslant, \ldots\right.$, $\geqslant z_{d}^{\downarrow}$. The interest of this work in the majorization concept comes from a theorem which states that a bipartite pure state $|\psi\rangle$ may be transformed to another pure state $|\phi\rangle$ by LOCC if and only if $\lambda_{\psi}<\lambda_{\phi}$, where $\lambda_{\psi}, \lambda_{\phi}$ are the vectors of (square) coefficients of the Schmidt decomposition of the states $|\psi\rangle$, $|\phi\rangle$, respectively. LOCC adds to those quantum operations effected only locally the possibility of classical communication between spatially separated parts of the system. According to this criterion, it would be possible, in principle, to obtain a singlet spin state $|\phi\rangle$ beginning with a momentum entangled state $|\psi\rangle$ whenever $\lambda_{\psi}<\lambda_{\phi}$.

The possibility of obtaining a singlet spin state from a momentum-entangled state can be extended to a more efficient situation: the possibility of distillation of entanglement. This idea consists on obtaining multiple singlet states beginning with several copies of a given pure state $|\psi\rangle$. The distillable entanglement of $|\psi\rangle$ consists in the ratio $n / m$, where $m$ is the number of copies of $|\psi\rangle$ we have initially, and $n$ the number of singlet states we are able to obtain via LOCC acting on these copies. It can be shown [6] that for pure states the distillable entanglement equals the entropy of entanglement $S$. Thus, in the continuous case (infinitedimensional Hilbert space), the distillable entanglement is 
not bounded from above, because neither is S. According to this, the larger the entanglement in momenta the more singlet states could be obtained with LOCC.

To illustrate the possibility of entanglement transfer with a specific example, we consider a momentum-entangled state for two distinguishable fermions

$$
|\psi\rangle=\frac{1}{\sqrt{2}}\left[\psi_{1}^{(1)}(p) \psi_{1}^{(2)}(q)+\psi_{2}^{(1)}(p) \psi_{2}^{(2)}(q)\right]|\uparrow \uparrow\rangle .
$$

This state has associated a vector $\lambda_{\psi}^{\downarrow}=(1 / 2,1 / 2,0,0, \ldots)$. On the other hand, the singlet state

$$
|\phi\rangle=\psi_{1}^{(1)}(p) \psi_{1}^{(2)}(q) \frac{1}{\sqrt{2}}(|\uparrow \downarrow\rangle-|\downarrow \uparrow\rangle)
$$

has associated a vector $\lambda_{\phi}^{\downarrow}=(1 / 2,1 / 2,0,0, \ldots)$.

These vectors obey $\lambda_{\psi}<\lambda_{\phi}$, and thus the state entangled in momenta may be transformed into the state entangled in spins via LOCC.

\section{Kinematical transfer and Lorentz boosts}

Another approach to the study of entanglement transfer between momentum and spin degrees of freedom is the kinematical one. In fact, the Lorentz transformations may entangle the spin and momentum degrees of freedom. To be more explicit, and following the notation of Ref. [12], we consider a certain bipartite pure wave function $g_{\lambda \sigma}(\mathbf{p}, \mathbf{q})$ for two spin- $-\frac{1}{2}$ fermions, where $\lambda$ and $\sigma$ denote, respectively, the spin degrees of freedom of each of the two fermions, and $\mathbf{p}$ and $\mathbf{q}$ the corresponding momenta. This would appear to an observer in a Lorentz transformed frame as

$$
g_{\lambda \sigma}(\mathbf{p}, \mathbf{q}) \stackrel{\Lambda}{\Lambda} \sum_{\lambda^{\prime} \sigma^{\prime}} U_{\lambda \lambda^{\prime}}^{\left(\Lambda^{-1} \mathbf{p}\right)} U_{\sigma \sigma^{\prime}}^{\left(\Lambda^{-1} \mathbf{q}\right)} g_{\lambda^{\prime} \sigma^{\prime}}\left(\Lambda^{-1} \mathbf{p}, \Lambda^{-1} \mathbf{q}\right)
$$

where

$$
U_{\lambda \lambda^{\prime}}^{(\mathbf{p})} \equiv D_{\lambda \lambda^{\prime}}^{(1 / 2)}[R(\Lambda, \mathbf{p})],
$$

is the spin $-\frac{1}{2}$ representation of the Wigner rotation $R(\Lambda, \mathbf{p})$. The Wigner rotations of Eq. (B4) can be seen as conditional logical operators, which rotate the spin a certain angle depending on the value of the momentum. Thus, a Lorentz transformation will modify in general the entanglement between momentum and spin of each individual electron. We distinguish the following three cases.

(1) Product state in all variables. In this case,

$$
g_{\lambda \sigma}(\mathbf{p}, \mathbf{q})=g_{1}(\mathbf{p}) g_{2}(\mathbf{q})|\lambda\rangle|\sigma\rangle,
$$

and the entanglement at the rest reference frame is zero. Under a boost, the Wigner rotations of Eq. (B4) entangle the momentum of each fermion with its spin, and thus the entanglement momentum-spin grows [10].

(2) Entangled state spin-spin and/or momentummomentum. We consider now a state

$$
g_{\lambda \sigma}(\mathbf{p}, \mathbf{q})=f(\mathbf{p}, \mathbf{q})|\phi\rangle
$$

with $|\phi\rangle$ an arbitrary state of the spins, and $f(\mathbf{p}, \mathbf{q})$ an arbitrary state of the momenta. In this case, a Lorentz boost will entangle, in general, each spin with its corresponding momentum, and a careful analysis shows that the spin-spin entanglement never grows [12]. Of course, by applying the reversed boost the entanglement momentum-spin would be transferred back to the spin-spin one, and thus the latter would grow. This particular case shows that, for the state we considered in Sec. III, given by Eqs. (24), (25), and (27), the entanglement could not be transferred from momenta into spins via Lorentz transformations. Thus, the dynamical approach would be here more suitable.

(3) Entangled state momentum-spin. According to the previous theorem, the momentum-spin entanglement may be lowered, transferring part of the correlations to the spins, or increased, taking some part of the correlations from them. To our knowledge, there is not a similar result for the momentum, that is, whether the momentum entanglement can be preserved under boosts, or it suffers decoherence similarly to the spins, and part of it is transferred to the momentum-spin part. This is a very interesting question, which we will treat more deeply in future works.
[1] A. Einstein, B. Podolsky, and N. Rosen, Phys. Rev. 47, 777 (1935).

[2] J. S. Bell, Physics (Long Island City, N.Y.) 1, 195 (1964).

[3] C. H. Bennett, G. Brassard, C. Crépeau, R. Jozsa, A. Peres, and W. K. Wootters, Phys. Rev. Lett. 70, 1895 (1993).

[4] L. K. Grover, e-print quant-ph/9704012; J. I. Cirac, A. K. Ekert, S. F. Huelga, and C. Macchiavello, Phys. Rev. A 59, 4249 (1999).

[5] A. K. Ekert, Phys. Rev. Lett. 67, 661 (1991).

[6] M. A. Nielsen and I. L. Chuang, Quantum Computation and Quantum Information (Cambridge University Press, Cambridge, England, 2000).

[7] A. Galindo and M. A. Martín-Delgado, Rev. Mod. Phys. 74, 347 (2002).
[8] B. -G. Englert and K. Wódkiewicz, Int. J. Quantum Inf. 1, 153 (2003).

[9] M. Czachor, Phys. Rev. A 55, 72 (1997).

[10] A. Peres, P. F. Scudo, and D. R. Terno, Phys. Rev. Lett. 88, 230402 (2002).

[11] P. M. Alsing and G. J. Milburn, Quantum Inf. Comput. 2, 487 (2002).

[12] R. M. Gingrich and C. Adami, Phys. Rev. Lett. 89, 270402 (2002).

[13] R. M. Gingrich, A. J. Bergou, and C. Adami, Phys. Rev. A 68, 042102 (2003)

[14] J. Pachos and E. Solano, Quantum Inf. Comput. 3, 115 (2003).

[15] H. Terashima and M. Ueda, Quantum Inf. Comput. 3, 224 (2003). 
[16] D. Ahn, H. J. Lee, Y. H. Moon, and S. W. Hwang, Phys. Rev. A 67, 012103 (2003).

[17] A. Peres and D. R. Terno, Rev. Mod. Phys. 76, 93 (2004).

[18] E. B. Manoukian and N. Yongram, Eur. Phys. J. D 31, 137 (2004).

[19] R. Grobe, K. Rząewski, and J. H. Eberly, J. Phys. B 27, L503 (1994).

[20] E. E. Salpeter and H. E. Bethe, Phys. Rev. 84, 1232 (1951).

[21] M. Gell-Mann and F. Low, Phys. Rev. 84, 350 (1951).

[22] L. Lamata and J. León, J. Opt. B: Quantum Semiclassical Opt. 7, 224 (2005).

[23] J. Schliemann, J. I. Cirac, M. Kuś, M. Lewenstein, and D.
Loss, Phys. Rev. A 64, 022303 (2001).

[24] K. Eckert, J. Schliemann, D. Bruß, and M. Lewenstein, Ann. Phys. 299, 88 (2002).

[25] G. Ghirardi and L. Marinatto, Fortschr. Phys. 52, 1045 (2004). [26] M. Moshinsky, Phys. Rev. 88, 625 (1952).

[27] I. S. Gradshteyn and I. M. Ryzhik, Table of Integrals, Series, and Products (Academic Press, Inc., Orlando, 1980), Eq. 7.374.8

[28] A. Ekert and P. L. Knight, Am. J. Phys. 63, 415 (1995).

[29] C. K. Law, I. A. Walmsley, and J. H. Eberly, Phys. Rev. Lett. 84, 5304 (2000).

[30] M. A. Nielsen, Phys. Rev. Lett. 83, 436 (1999). 